\title{
NEW CHARACTERIZATIONS OF VON NEUMANN REGULAR RINGS AND A CONJECTURE OF SHAMSUDDIN
}

\author{
Carl Faith
}

\begin{abstract}
A theorem of Utumi states that if $R$ is a right self-injective ring such that every maximal ideal has nonzero annihilator, then $R$ modulo the Jacobson radical $J$ is a finite product of simple rings and is a von Neuman regular ring. We prove two theorems and a conjecture of Shamsuddin that characterize when $R$ itself is a von Neumann ring, using a splitting theorem of the author on when the maximal regular ideal of a ring splits off.
\end{abstract}

\section{Introduction.}

Using splitting off theorems for the maximal Von Neumann regular ideal $M(R)$ of a ring $R$ in the author's paper [F], we verify a conjecture of Shamsuddin's as a consequence of the following:

Theorem 1. Let $R$ be a ring with Jacobson radical J. The f.a.e.c.'s:

(1) $R$ is von Neumann regular $(=V N R)$.

(2) $R$ is semiprime, $\bar{R}=R / J$ is VNR, $M(R)$ splits off, and $J$ is an annihilator (left or right) ideal.

As another consequence of $M(R)$ splitting we deduce:

Theorem 2. Let $R$ be a two-sided continuous (e.g., two-sided selfinjective) ring. Then, $R$ is VNR iff $R$ is a semiprime ring whose Jacobson radical is an annihilator ideal.

We now state: 
Shamsuddin's Conjecture. Let $R$ be a ring such that $R / J$ is a $V N R$ and a finite product of simple ideals $A_{1}, \ldots, A_{n}$. Then $R$ is VNR iff $R$ is a semiprime ring and $J$ is an annihilator ideal.

Proof of Theorem 1: The necessity if trivial. Conversely, by assumption, $R=M \times R_{2}$, where $M=M(R)$ and $R_{2}$ has zero regular ideal, that is, $M\left(R_{2}\right)=0$. Moreover, $J \subseteq R_{2}$. We shall show that $R_{2}=0$, and then $R=M(R)$ is von Neumann regular. Since $R$ is semiprime each nonzero ideal $I$ of $R$ satisfies

$$
{ }^{\perp} I \bigcap I=I^{\perp} \bigcap I=0
$$

where the exponent $\perp$ denotes the annihilator on the appropriate side. Clearly ${ }^{\perp} J \supseteq M$ hence ${ }^{\perp} J=M \oplus\left({ }^{\perp} J \bigcap R_{2}\right)$, hence assuming $J$ is a right annihilator, then

$$
J=\left({ }^{\perp} J\right)^{\perp}=M^{\perp} \bigcap\left({ }^{\perp} J \bigcap R_{2}\right)^{\perp} .
$$

Since ${ }^{\perp} J \bigcap R_{2}=\ell_{R_{2}}(J)$ is the left annihilator of $J$ in $R_{2}$, then

$$
\left({ }^{\perp} J \bigcup R_{2}\right)^{\perp}=\ell_{R_{2}}(J)^{\perp}=M+r \ell_{R_{2}}(J)
$$

where $R()$ denotes right annihilation in $R_{2}$. Using $(*)$ and the fact that $M^{\perp}=R_{2}$, then

$$
\begin{aligned}
J & =M^{\perp} \bigcap\left(M+r \ell_{R_{2}}(J)\right) \\
& =R_{2} \bigcap\left(M+r \ell_{R_{2}}(J)\right) .
\end{aligned}
$$

Since $R_{2} \bigcap M=0$, and $R_{2} \supseteq r \ell_{R_{2}}(M)$, then $(* *)$ implies that $J=$ $r \ell_{R_{2}}(J)$. If $R_{2} \neq 0$, then $J \neq R$, hence $\ell_{2}(J) \neq 0$. But $R_{2}$ is semiprime (along with $R$ ), hence $\ell_{2}(J) \bigcap J=0$ which shows that $L=\ell_{2}(J)$ embeds canonically in $\bar{R}_{2}=R_{2} / J$. Since

$$
\bar{R}=R / J=M \times\left(R_{2} / J\right)
$$

is VNR, then $\bar{R}_{2}=R_{2} / J$ is also VNR, and hence $L$ is a VNR ideal. This shows that $L$ is an VNR ideal of $R_{2}$ contrary to the fact that $M\left(R_{2}\right)=0$, that is $R_{2}$ has no nonzero regular ideals. This contradiction shows that $R_{2}=0$, and hence $R=M(R)$ is VNR as asserted.

Proof of Conjecture: By Theorem 2 of [F] (and its Corollary), $M=$ $M(R)$ splits off in $R$ as a ring direct summand iff the canonical image $\bar{M}$ of $M$ in $\bar{R}=R / J$ splits off as a right or left ideal. Under the assumption 
of the conjecture, the only ideals of $\bar{R}$ are the finite products of a subset of $\left\{0, A_{1}, \ldots, A_{n}\right\}$ without repetitions, and all of these are necessarily ring direct summands of $\bar{R}$. Thus $\bar{M}$ splits off in $\bar{R}$, hence $M$ splits off in $R$, so Theorem 2 applies to prove that $R$ is VNR.

Proof of Theorem 2: By the main theorem of $[\mathbf{F}], M(R)$ splits off in any two-sided continuous (eg. self-injective) ring, so Theorem 1 applies.

We conclude with another corollary as applications of two theorems of Utumi. In

Corollary 3. If $R$ is a right self-injective ring in which maximal ideals have nonzero annihilators, then $R$ is semiprime iff $R$ is VNR. In this case, $R$ is a finite product of simple rings.

Proof: By Theorem 2.3 of $[\mathbf{U} \mathbf{1}], R$ is a finite product of simple rings, and $J$ is an annihilator ideal. Moreover, $R / J$ is VNR by Theorem 4.8 of [U2]. Thus the truth of Shamsuddin's conjecture completes the proof.

Acknowledgement. Professor Ahmad Shamsuddin made this conjecture during his visit to Rutgers University in September 1994. I have to thank him also for the reference [U1] used in the proof of Corollary 3.

\section{References}

[F] C. FAith, The maximal regular ideal of self-injective and continuous rings splits off, Arch. Math. 4 (1985), 511-521.

[U1] Y. Utumi, Self-injective rings, J. Algebra 6 (1967), 59-64.

[U2] Y. Utumi, Continuous rings and self-injective rings, Trans. Amer. Math. Soc. 118 (1965), 158-173.

Department of Mathematics

Rutgers University

Hill Center for Mathematical Sciences

Busch Campus

New Brunswick, New Jersey 08903

U.S.A.
Author's mailing address:

199 Longview Drive

Princeton, NJ 08540

U.S.A.

Primera versió rebuda el 5 de Desembre de 1995, darrera versió rebuda el 10 d'Abril de 1996 